\title{
The mass distribution in Spiral galaxies
}

\author{
Paolo Salucci \\ SISSA \\ Via Beirut, 2-4, 34100 Trieste, Italy \\ email: salucci@sissa.it
}

\begin{abstract}
In the past years a wealth of observations has allowed us to unravel the structural properties of the Dark and Luminous mass distribution in spirals. As result, it has been found that their rotation curves follow, out their virial radius, an Universal function (URC) made by two terms: one due to the gravitational potential of a Freeman stellar disk and the other due to that of a dark halo. The importance of the latter is found to decrease with galaxy mass. Individual objects reveal in detail that dark halos have a density core, whose size correlates with its central value. These properties will guide $\Lambda$ CDM Cosmology to evolve to match the challenge that observations presently pose.
\end{abstract}

Keywords. dark matter, galaxies: formation, galaxies: kinematics and dynamics

\section{Introduction}

Rotation curves (hereafter RC) of disk galaxies do not show any Keplerian fall-off and do not match the distribution of the stellar (plus gaseous) matter. As a most natural explanation, this implies an additional invisible mass component (Rubin et al. 1980; Bosma 1981, Persic \& Salucci, 1988) that becomes progressively more conspicuous at outer radii and for the less luminous galaxies (e.g.: Persic \& Salucci, 1988; Broeils, 1992). The distribution of matter in disk systems has become a crucial benchmark for the present understanding of the process of galaxy formation. Time is ripe that we can address, with the help of the available observational scenario, crucial questions: i) has the DM an universal distribution reflecting its very Nature? ii) how and why the dark-to-luminous mass ratio and other dark and luminous physical quantities vary in objects of different mass?

It is well known that numerical simulations performed in the framework of the currently favored theory of structure formation, $\Lambda$ Cold Dark Matter, predict a well-defined density profile for the virialized halos (Navarro, Frenk and White, 1996). Furthermore, the mechanism of galaxy formation, as currently understood, involves the cooling and the condensation of pristine HI gas inside the gravitational potential well of DM halos, the transformation of part of this gas into stars and finally the feedback of the latter on the former, through SN explosions. It is widely accepted that the mass distribution of spiral galaxies, we can derive from observations, bears the imprint of the Nature and the cosmological history of the DM, its interaction with the LM and it highlights the astrophysical processes that have turned (some of) the pristine infalling gas into a stellar disk.

It is worth to define the Dark Matter in the following way: let us set $M(R)$ the mass distribution of the gravitating matter and $M_{L}(R)$ that of the baryonic "luminous" matter, both obtained from observations. Realized that these distributions do not match, i.e. $d \log M(r) / d \log R>d \log M_{L} / d \log R$, we introduce a dark mass component with mass 

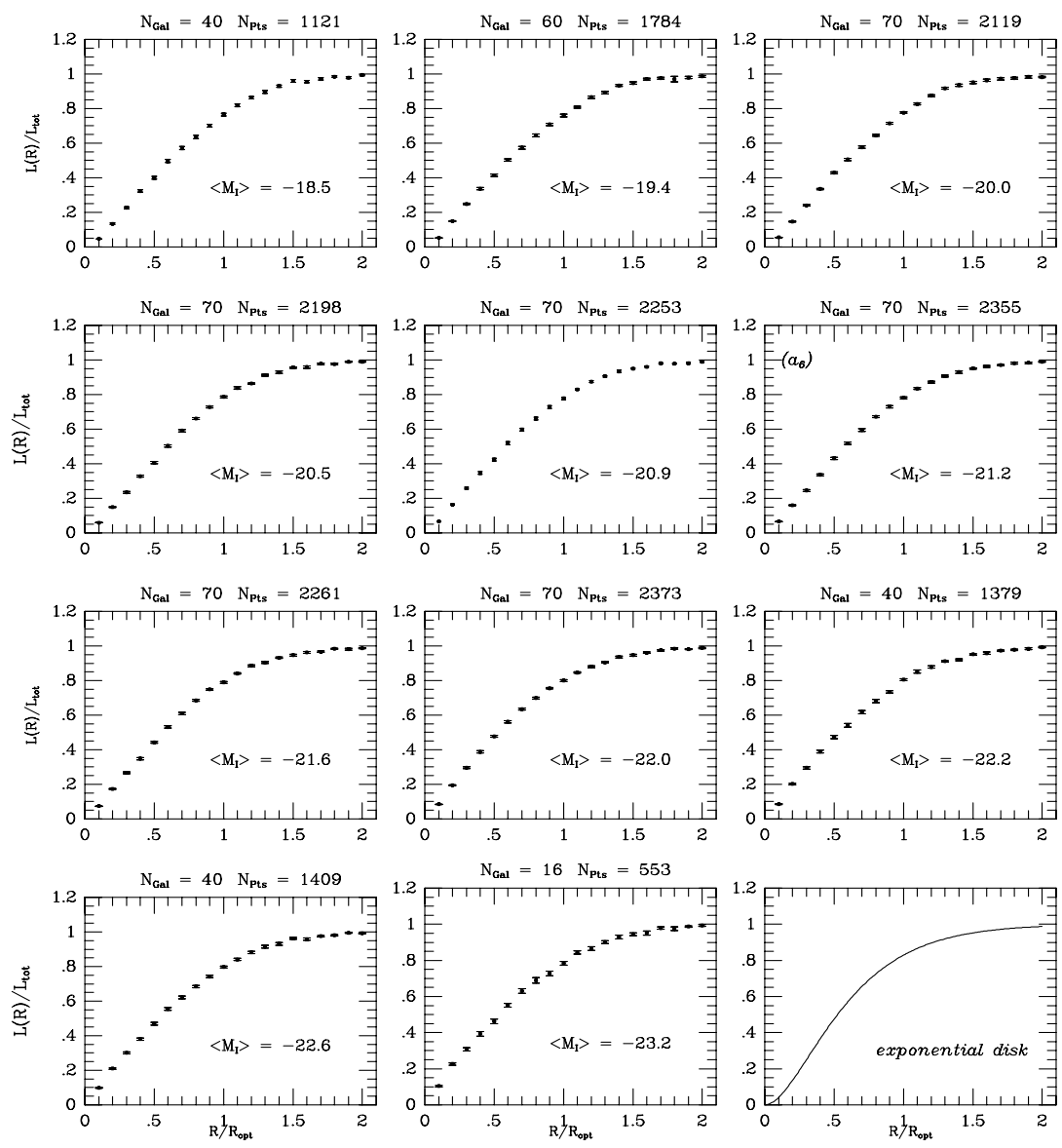

Figure 1. The coadded luminosity profiles $L\left(R / R_{o p t}\right) / L_{t o t}=\int_{0}^{R / R_{o p t}} I\left(r^{\prime}\right) r^{\prime} d r^{\prime} / L_{t o t}$.

profile $M_{h}(R)$ such that:

$$
\frac{d \log M(R)}{d \log R}=\frac{M_{L}(R)}{M(R)} \frac{d \log M_{L}}{d \log R}+\frac{M_{h}(R)}{M(R)} \frac{d \log M_{h}}{d \log R}
$$

With this definition it is immediate that the study of the DM phenomenon involves the slope of the RC rather than its amplitude and must start from a proper knowledge of the luminous matter distribution. In spirals, this has three components: a stellar bulge, a thin stellar disk and an extended thin HI disk. Within the aim of this paper the latter component plays little role and it will be neglected unless we consider individual HI-rich objects. The first component, instead, it is important in early type spirals, not considered here and then we redirect the reader to Noordermeer et al. (2007).

The luminous matter in spirals is distributed in thin disk with surface luminosity (Freeman, 1970):

$$
I(R)=I_{0} e^{-R / R_{D}}
$$

with $R_{D}$ the disk scale-length; $I_{0}$ the central value; it is useful to define $R_{o p t} \equiv 3.2 R_{D}$ as the "size" of the stellar disk, whose luminosity is $L_{t o t}=2 \pi I_{0} R_{D}^{2}$. In Fig. 1 we plot the coadded (I-band) light profiles of 616 late types spirals arranged in 11 luminosity bins as 


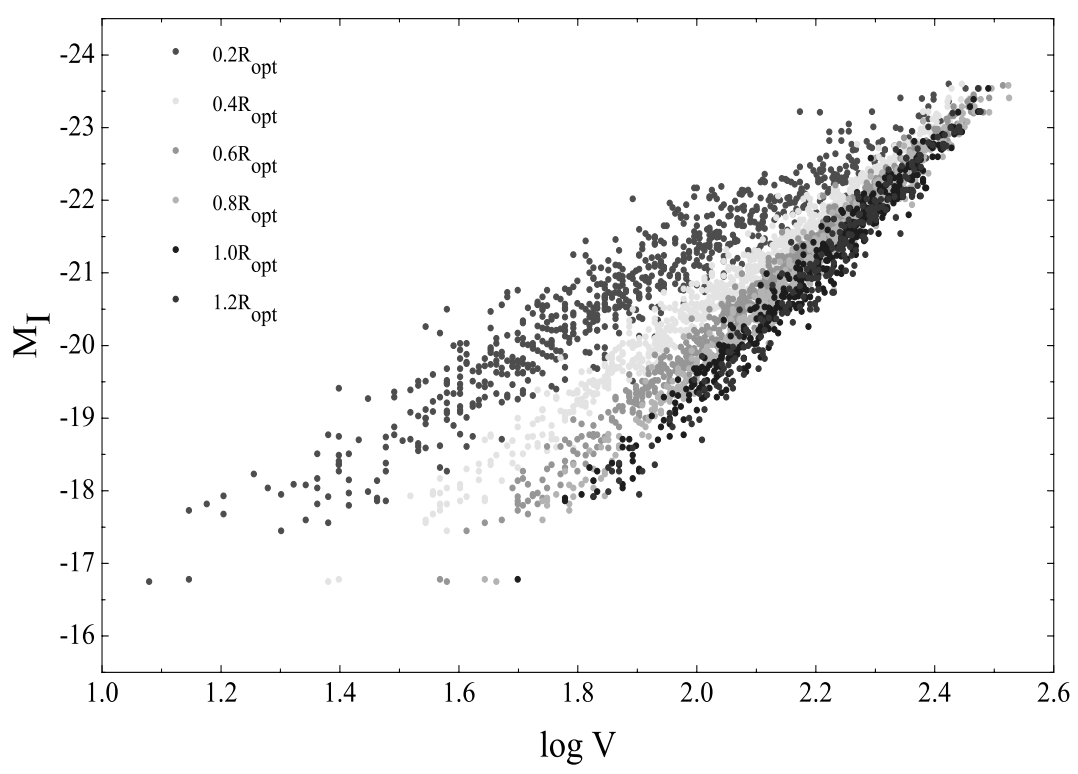

Figure 2. The Radial TF relation.

a function of radius, expressed in units of optical radius. We realize that the light profile does not depend on galaxy luminosity and it is well represented by a Freeman profile.

Although the mass modelling of some individual object may require to consider nonexponential stellar disks, eq (1.2) well represents the typical distribution of stars in late type spirals. Similarly, the moderate radial variations of the stellar mass-to-light ratios in spirals will be neglected as they are irrelevant for the present issues (Portinari \& Salucci, 2007).

Finally we assume flat cosmology with matter density parameter $\Omega_{M}=0.27$ and Hubble constant $H_{0}=71 \mathrm{~km} \mathrm{~s}^{-1} \mathrm{Mpc}^{-1}$, at the present time, the size $R_{v i r}$ and the mass $M_{v i r}$ of a virialized halo are related by:

$$
R_{v i r}=259\left(\frac{M_{v i r}}{10^{12} \mathrm{M}_{\odot}}\right)^{1 / 3} \mathrm{kpc}
$$

\section{The RC's as gravitational field tracer}

In self-gravitating systems in full centrifugal equilibrium, the circular velocity $V$ at a radius $R$ is linked to the gravitational field $\Phi$ by $V^{2} / R=R d \Phi / d R$. In spirals, we measure the rotation velocity $V_{\text {rot }}$, i.e. the projection on the line of sight of the tangential velocity in cylindric coodinates. Let us notice that: $V_{\text {rot }}=V+V_{n a}$, where the latter defines non-axysimmetric motions unrelated to the central potential. $V_{\text {rot }}$ measures the gravitational field only when the latter motions are negligible. The discovery, at radii $R_{n} \equiv(n / 5) R_{o p t}$, of the Radial Tully-Fisher relation (Yegorova \& Salucci, 2007), i.e. the discovery of an ensemble of TF-like, statistically independent low scatter relationships between the galaxy absolute magnitude and the rotation velocity (see Fig. 2) :

$$
M_{\text {band }}=a_{n} \log V_{n}+b_{n},
$$

where $V_{n} \equiv V_{\text {rot }}\left(R_{n}\right)$, and $a_{n}, b_{n}$ are the slope and zero-point of the relations, indicates that the RC's are good gravitational field tracer. More specifically, the fact that in any 


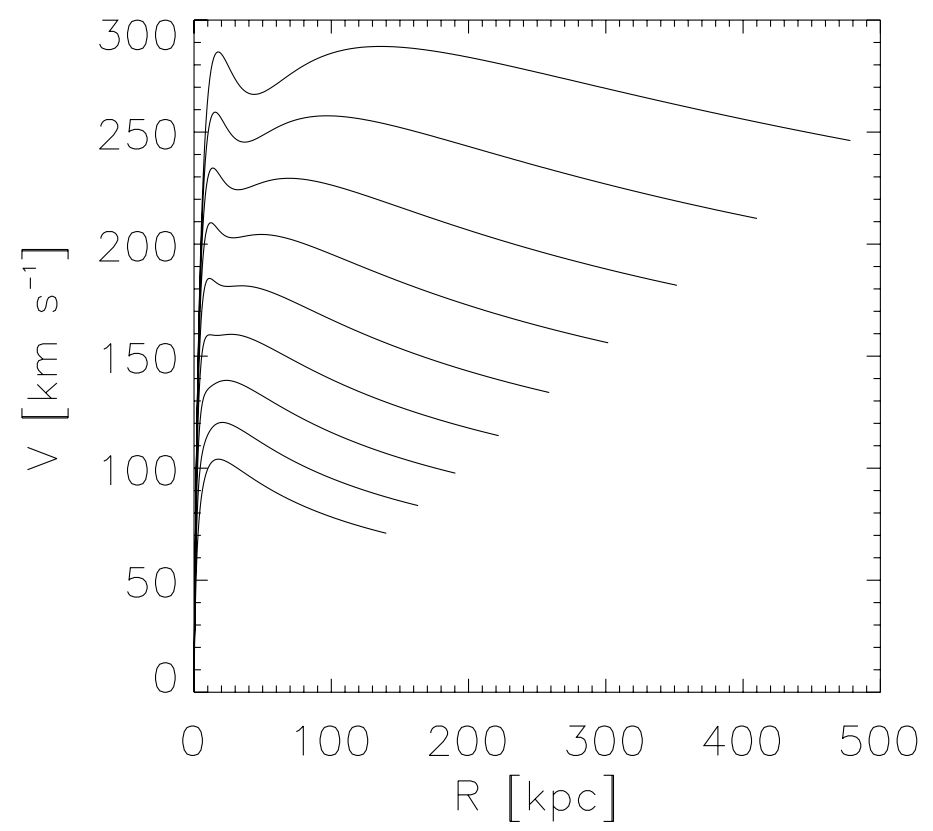

Figure 3. The Universal Rotation Curve with the radial coordinate in physical units. Each curve corresponds to $M_{v i r}=10^{11} 10^{n / 5} \mathrm{M}_{\odot}$, with $n=1 \ldots 9$ from the lowest to the highest curve.

object and at any radius, the rotation velocity can be predicted just by the galaxy luminosity implies that $V_{n a}$ is generally negligible. A similar conclusion is reached when we reproduce the innermost regions of spirals RC's with mass models with the luminous matter alone that leave no space both for DM and for significant non circular motions (Ratnam et al., 2000).

\section{The Universal Rotation Curve}

The kinematical properties of Sb-Im spirals led to the Universal Rotation Curve (URC) paradigm, pioneered in Persic and Salucci (1991) and set in Persic, Salucci \& Stel (1996, hereafter PSS). We can state that (see also Salucci et al., 2007) the RCs can be generally represented out to their virial radius by $V_{U R C}(R ; P)$, i.e. by a Universal function of radius, tuned by some galaxy property $P$, such as the luminosity or the virial mass that serves as galaxy identifier. The radius can be measured in three coordinate systems $R, R / R_{D}, R / R_{v i r}$ : the physical coordinate, and coordinates focused on the luminous and the dark matter distributions respectively.

The data used to build the URC are 1) 616 RCs that are first arranged in 11 luminosity intervals spanning the whole $I$-band luminosity range $-16.3<\mathrm{M}_{I}<-23.4$, with each luminosity bin having $\sim 1500$ velocity measurements, and then coadded by arranging the latter in radial bins of size $0.3 R_{D}$. This builds 11 synthetic curves $V_{\text {coadd }}\left(R / R_{\text {opt }}, M_{I}\right)$ out to $\sim 4 R_{D}$ (see PSS), free from most of the observational errors and non-axisymmetric disturbances present in individual RCs. These RC's result very smooth and with a very small intrinsic variance, but showing a very strong luminosity dependence (see also Catinella et al., 2006) b) the empirical relationship between RC slope at $2 R_{o p t}$ and $\log V_{\text {opt }}$ (see 


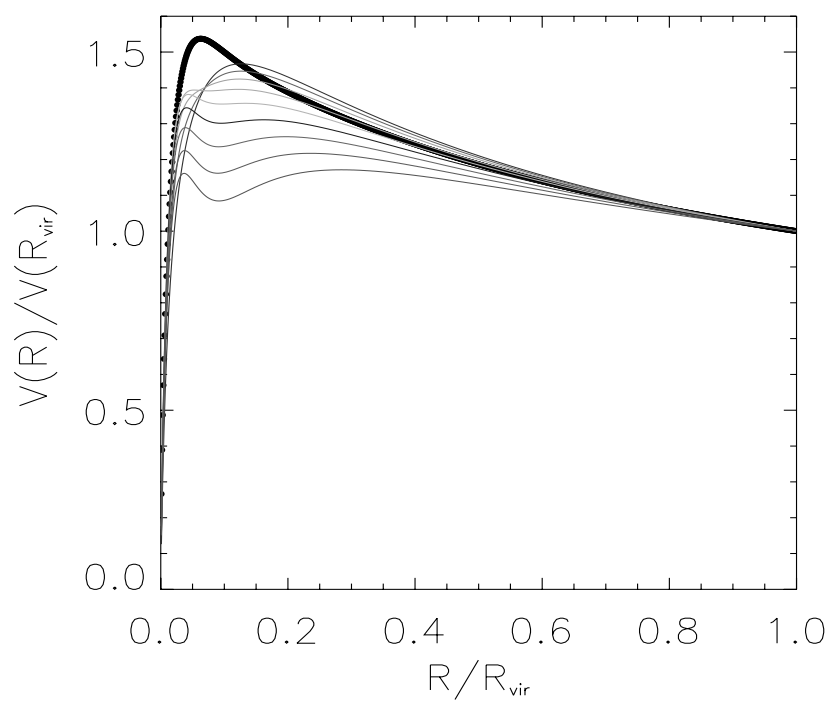

Figure 4. The Universal Rotation Curve, normalized at its virial value as a function of normalized "dark" radius $R / R_{v i r}$. Each curve, from the highest to the lowest, corresponds to $M_{v i r}$ as in Fig. 2. The bold line is the NFW velocity profile.

PSS) c) the halo virial velocity $V_{v i r} \equiv\left(G M_{v i r} / R_{v i r}\right)^{1 / 2}$, obtained from the disk mass vs. virial mass relationship (see Shankar et al., 2006).

The URC paradigm, i.e. the idea according to which halo mass (or the disk mass), eventually involving few other quantities, "determines" at any radii the circular velocity of any spiral within an error that is typically much smaller than the true variations that the RC shows in galaxies and among galaxies is implemented by the URC function, an analytical Curve we construct as the sum in quadrature of the disk and halo contributions to the circular velocity, meant to be the observational counterpart of the NFW velocity profile.

Then, we set: $V_{U R C}^{2}=V_{U R C D}^{2}+V_{U R C H}^{2}$. From (1.2) the disk term is:

$$
V_{U R C D}^{2}(x)=\frac{1}{2} \frac{G M_{D}}{R_{D}}(3.2 x)^{2}\left(I_{0} K_{0}-I_{1} K_{1}\right)
$$

where $x=R / R_{\text {opt }}$ and $I_{n}$ and $K_{n}$ are the modified Bessel functions computed at $1.6 x$.

For the DM term we assume that the dark halo follows a Salucci \& Burkert (2000) profile, a cored distribution that can converges to a NFW one at outer radii

$$
\rho(R)=\frac{\rho_{0} r_{0}^{3}}{\left(R+r_{0}\right)\left(R^{2}+r_{0}^{2}\right)},
$$

$r_{0}$ is the core radius and $\rho_{0}$ the central density density. Then:

$$
V_{U R C H}^{2}(R)=6.4 G \frac{\rho_{0} r_{0}^{3}}{R}\left\{\ln \left(1+\frac{R}{r_{0}}\right)-\tan ^{-1}\left(\frac{R}{r_{0}}\right)+\frac{1}{2} \ln \left[1+\left(\frac{R}{r_{0}}\right)^{2}\right]\right\} .
$$

Then, the URC function, has three free parameters $\rho_{0}, r_{0}, M_{D}$ that are obtained from fitting $V_{\text {coadd }}$ and the other data specified above.

In Fig. 3 we show $V_{U R C}\left(R ; M_{v i r}\right)$, the URC with the radius expressed in physical units and the objects identified by the halo virial mass; each line refers to a given halo mass in the range $10^{11} M_{\odot}-10^{13} M_{\odot}$; the halo mass determines both the amplitude and the shape of the curve. Note the contribution of the baryonic component, negligible for small masses but increasingly important in the larger structures. In Fig. 4 we frame the URC 

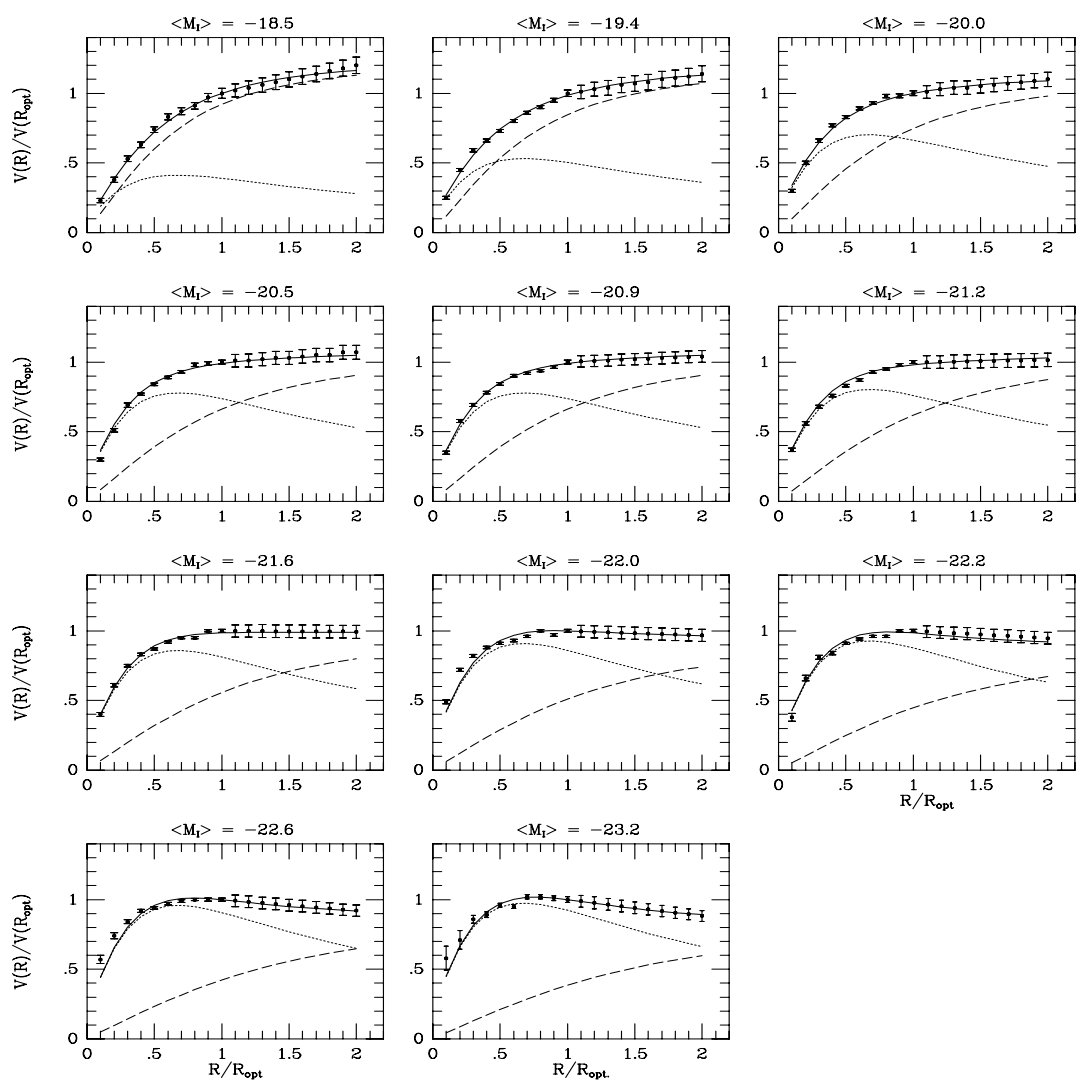

Figure 5. Best disk-halo fits to the Universal Rotation Curve (dotted/dashed line: disc/halo).

from a full DM perspective by plotting $V_{U R C}\left(R / R_{v i r} ; M_{v i r}\right), R / R_{v i r}$ is the radial "dark" coordinate, and the URC is normalized by $V_{v i r} \propto M_{v i r}^{1 / 3}$.

The URC shows that the DM halos and stellar disks are both self-similar, but the whole system is not, likely due to the baryons collapse that has have broken it in the innermost $30 \%$ of the halo size.

Let us notice that the RC's of spirals are critically not flat: their RC slopes take all of sort of values from that of solid-body system (i.e. +1 ) to almost that of a Newtonian point-mass (i.e. $-1 / 2$ ). The maximum of the $\mathrm{RC}$ occurs at very different radii, for galaxies of different mass, viz. at $\simeq 2 R_{D}$ for the most massive objects and at $\sim 10 R_{D}$ for the least massive ones.

\section{The mass distribution in Spirals}

The existence of systematical properties of the mass distribution in spirals was first claimed by Persic and Salucci (1988) and then successively confirmed by independent works (Broeils, 1992; PSS; Rhee, 1997; Swaters, 1999). However, until now it has been considered by current theory of galaxy formation only in a limited way. Likely, this is a consequence of the theoretical prejudice that the cosmological galaxy formation process did not leave relevant features in the distribution of matter in galaxies and of 

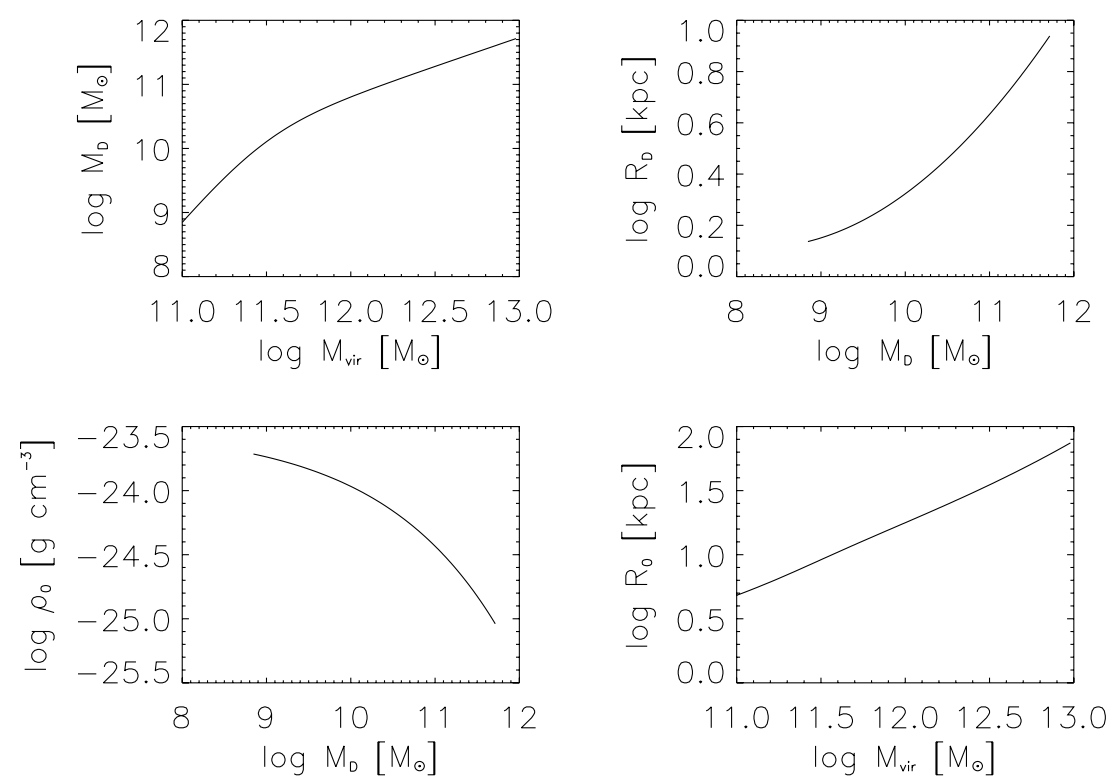

Figure 6. The structural parameters of the mass distribution.

the difficulties that simulations have in "reproducing" reasonable disk systems. However, after Salucci et al. (2007), we believe that, in order to understand the whole process of structure formation in the Universe, we must take into account the rich scenario of the dark-luminous interplay occurred in galaxies.

The mass distribution in Spirals is obtained by mass modelling two very different and complementary kinematical set of data i) a large number of individual RC's of objects of different luminosity and b) the URC (see Fig. 5), that, let us remind, originates from coadded RC's; noticeably, the results obtained from these two different sets of data are very similar, indicating so that they are robust and reliable. A clear scenario of the mass distribution emerges, see Fig. 6:

- The stellar disk dominates the galaxy's inner region out to the radius $R_{I B D}$ at which the DM halo contribution starts to take over the stellar one. This sets the properties of the Radial Tully Fisher relation and yields to the paradigm of the Inner Baryon Dominance: the inner portions of the (observed) RC that can be accounted by the stellar matter alone are indeed saturated by this component.

- At any radii, smaller lower luminosity objects have more progressively more proportion of dark matter i.e. a larger dark-to-stellar mass ratio. In detail, the disk mass is $\propto M_{v i r}^{2}$ at small virial masses, $M_{v i r}=10^{11} M_{\odot}$ and $\propto M_{v i r}$ at larger masses, $M_{v i r}=$ $10^{13} M_{\odot}$. The baryonic fraction is always much smaller than $\Omega_{b} / \Omega_{\text {matter }} \simeq 1 / 6$ i.e. the cosmological value and it ranges between $7 \times 10^{-3}$ to $5 \times 10^{-2}$ in line with is the wellknown evidence that SN explosions have removed (or made never condense) a very large fraction of the original HI material.

- Smaller spirals are denser, with the central density spanning 2 order of magnitudes over the mass sequence of spirals. If this reflects the background density at the formation time, it indicates that the present day population of spirals has been hierarchically formed from $z=5$ to $z=1$, and, since then, it has experienced very little merging.

- The structural parameters of the mass distribution, $\rho_{0}, M_{D}, M_{h}, r_{0}$ are remarkably all related among themselves, see figure (4) and fig. (11) of PSS. Notice that, although 


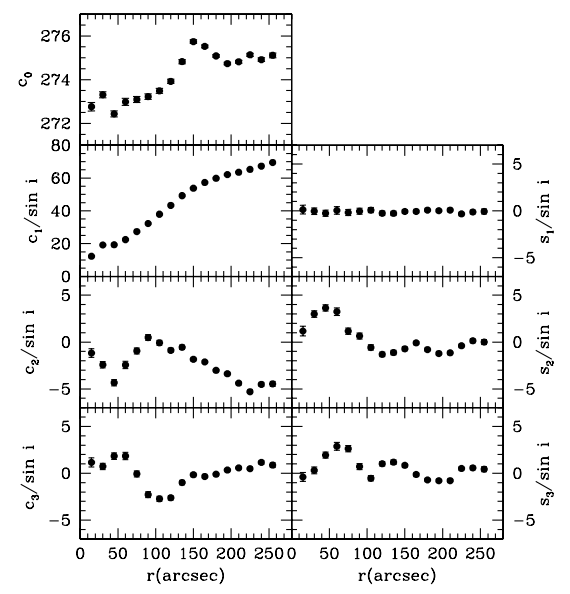

Figure 7. Harmonic decomposition of the 2D RC of DDO 47.

this evidence is connected with the URC paradigm, however it is not directly implied by it nor it implies it.

- The stellar mass-to-light ratio is found to lie between 0.5 and 4 . The values of disk masses derived as above agree very well with those obtained by fitting their SED with spectro-photometric models (Salucci, Yegorova, Drory, 2007)

- The HI component is almost always below the kinematical detectably. However, in low mass systems it cannot be neglected in the baryonic budget since it is more prominent than the stellar disk.

\section{The core-cusp issue}

A fundamental prediction of the cosmological $(\Lambda)$ Cold Dark Matter simulations is that virialized dark matter halos have an universal spherically averaged density profile $\rho_{C D M}(R)$ (Navarro, Frenk \& White, 1997)

$$
\rho_{C D M}(R)=\frac{\rho_{s}}{\left(R / r_{s}\right)\left(1+R / r_{s}\right)^{2}}
$$

where $\rho_{s}$ and $r_{s}$ are strongly correlated (e.g. Wechsler et al., 2002), we have: $r_{\mathrm{s}} \simeq$ $8.8\left(\frac{M_{v i r}}{10^{11} \mathrm{M}_{\odot}}\right)^{0.46} \mathrm{kpc}$ that sets the region of the inner cusp. The above profile converges, at small radii, to a power-law of index -1 , although, according to higher resolution simulations, the actual value of the index reaches about -1.35 (e.g. Moore et al. 1999, Navarro et al. 2004).

The CDM predictions can be confronted with observations: RC's of disk galaxies, in fact, probe the crucial region $0.1 r_{s} \leqslant r \leqslant 2 r_{s}$. Flores \& Primack (1994), Moore (1994) claimed, for some dwarfs, a tension between the kinematical data and the predictions of simulations: DM halos seemed to prefer cored density distributions rather than cuspy ones (see also van den Bosch and Swaters, 2001; Weldrake et al. 2003).

The importance of the issue, that concerns the very nature of dark matter, and the fact that these early results were questioned on several aspects, has triggered new investigations characterized by the study of few proper test-cases but with higher quality kinematical data and by means of a properly devised analysis (Gentile et al., 2004). These improvements were necessary: to obtain reliable DM profiles requires extended, regular, 


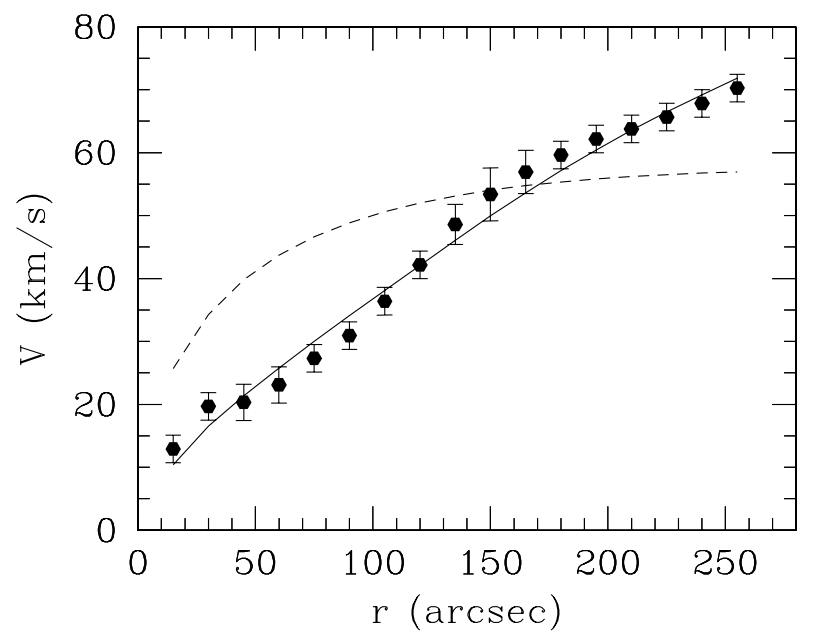

Figure 8. DDO 47 rotation curve (filled circles) best-fitted by Burkert halo + stellar disk (solid line) and by NFW halo + stellar disk (dashed line) mass models. The amplitude of non-circular motions is $<5 \mathrm{~km} / \mathrm{s}$.

homogeneous RC's reliable up to their second derivative and free from deviations from the axial symmetry. Then, up to now, few tenths of objects have qualified to undergo such critics-free investigation (e.g. the list in Donato et al., 2004; Simon et al., 2005; Gentile et al., 2005; 2007; de Blok, 2007).

In all these cases data and simulations were found in plain disagreement on three different aspects: the best-fit disk + NFW halo mass model a) fits the RC poorly and it implies b) an implausibly low stellar mass-to-light ratio and c) an unphysical high halo mass.

As an example, it is worth to discuss in detail the case of the nearby dwarf galaxy DDO 47 (Gentile et al. 2005). The HI observations have adequate resolution and sensitivity, showing that the HI 2-D kinematics is very regular, with a well-behaved velocity field. The observed velocity along the line of sight $V_{\text {los }}$ has been decomposed in terms of harmonic coefficients: $V_{\text {los }}=c_{0}+\sum_{j=1}^{n}\left[c_{j} \cos (j \psi)+s_{j} \sin (j \psi)\right]$ where $\psi$ is the azimuthal angle, $c_{0}$ is the systemic velocity, $c_{1}$ is the rotation velocity (see Schoenmakers et al., 1997); it is found that the coefficients $s_{1}, s_{3} j_{2}$ have a small amplitude (see Fig. 7) that excludes significant global elongation and lopsidedness of the potential and detects non-circular motions at a level of $3 \mathrm{~km} \mathrm{~s}^{-1}$ and with amplitude and radial profile very different from that necessary to hide a cuspy density distribution in the observed RC. The RC mass modelling, shown in Fig. (8), finds that the DDO 47 dark halo has a core radius of about $7 \mathrm{kpc}$ and a central density $\rho_{0}=1.4 \times 10^{-24} \mathrm{~g} \mathrm{~cm}^{-3}$, i.e. a much shallower distribution than that predicted by the NFW profile.

In all cases studied up to date a serious data-prediction discrepancy emerges, that becomes definitive when we remind that the actual $\Lambda C D M$ halo profiles are steeper than the standard NFW ones considered here and that the baryonic adiabatic collapse has likely contracted them further. On the other hand, this discrepancy found in a relatively small number of objects cannot be extended to the whole population of spirals, which is still not sufficiently investigated. Let us stress that there is not shortage of proposals aimed to explain the "density core phenomenon" even within $\Lambda$ CDM scenario (e.g. Tonini et al. 2007). 


\section{Conclusions}

The distribution of luminous and dark matter in galaxies shows amazing properties and a remarkable systematics that make it as one of the hottest cosmological issues. There is no doubt that this emerging observational scenario will be decisive in guiding how the $\Lambda C D M$-based theory of galaxy formation must evolve to meet the challenge that the observational data are posing.

\section{References}

Bosma, A. 1981, AJ, 86, 1791

Broeils, A. H. 1992, Ph.D. thesis, Groningen University

Catinella, B., Giovanelli R. \& Haynes, M. P., 2006, ApJ. 640, 751

Donato, F., Gentile, G. \& Salucci, P., 2004, MNRAS, 353, 17

de Blok, W. J. G. 2007, this book

Freeman, K. C. 1970, ApJ, 160, 811

Flores, R. \& Primack, J. R., 1994, ApJ, 427, L1

Gentile, G., Burkert, A., Salucci, P., Klein, U. \& Walter, F. 2005, ApJ, 634, L145

Gentile G., Salucci P., Klein U., Vergani D. \& Kalberla P., 2004, MNRAS, 351, 903

Gentile G., Salucci P., Klein U. \& Granato G.L., 2007, MNRAS, 375, 199

Moore, B., 1994, Nat., 370, 629

Moore, B., Quinn, T., Governato, F., Stadel, J. \& Lake, G., 1999, MNRAS, 310, 1147

Noordermeer E., van der Hulst J. M., Sancisi R., Swaters R. S. \& van Albada T.S., 2007, MNRAS, 376, 1513

Navarro J. F., et al., 2004, MNRAS, 349, 1039

Navarro, J. F., Frenk, C.S., \& White, S. D. M. 1996, ApJ, 462, 563

Persic, M. \& Salucci, P. 1988, MNRAS, 234, 131

Persic M. \& Salucci P., 1991, ApJ, 368, 60

Persic, M., Salucci, P. \& Stel, F. 1996, MNRAS, 281, 27 (PSS)

Portinari, L. \& Salucci, P. 2007, submitted

Rhee, M-H 1997, Thesis, Groningen University.

Ratnam C., Salucci P. \& 2000, NewA, 5, 427

Rubin, V. C., Ford, W. K., Jr. \& Thonnard, N. 1980, ApJ, 238, 471

Salucci P. \& Burkert A., 2000, ApJ, 537, L9

Salucci P., Lapi A., Tonini C., Gentile G., Yegorova I. \& Klein U., 2007, MNRAS, 378, 41

Salucci, P, Yegorova I. A. \& Drory, N., 2007, submitted

Shankar, F., Lapi, A., Salucci, P., De Zotti, G. \& Danese, L. 2006, ApJ, 643, 14

Schoenmakers, R. H. M., Franx, M. \& de Zeeuw, P. T., 1997, MNRAS, 292, 349

Simon, J. D., Bolatto, A. D., Leroy, A., Blitz, L. \& Gates, E. L. 2005, ApJ, 621, 757

Swaters, 1999, Ph.D. Thesis, Groningen University

Tonini C., Lapi A. \& Salucci P., 2006, ApJ, 649, 591

van den Bosch, F. C. \& Swaters, R.A., 2001 MNRAS, 325, 1017

Yegorova I. A. \& Salucci P., 2007, MNRAS, 377, 50

Wechsler, R. H., Bullock, J. S., Primack, J. L., Kravtsov, A. V. \& Dekel, A., 2002, ApJ, 568, 52

Weldrake, D. T. F., de Blok, W. J. G. \& Walter, F. 2003, MNRAS, 340, 12 